\title{
ON THE LATTICE OF ALL JOIN-ENDOMORPHISMS OF A LATTICE
}

\author{
G. GRÄTZER AND E. T. SCHMIDT
}

A many-one correspondence $\Theta$ of the lattice $L$ into itself is called a join-endomorphism, if it satisfies

$$
\Theta(x \cup y)=\Theta x \cup \Theta y
$$

for all $x, y \in L$.

It is easily shown that every join-endomorphism is an isotone correspondence (i.e. $x \geqq y$ implies $\Theta x \geqq \Theta y$ ). It is also easy to see that the set $I$ of antecedents of 0 under any join-endomorphism is an ideal. (For these and other facts used in the sequel we refer to the textbook of G. Birkhoff, Lattice theory, rev. ed., New York, 1948, henceforth cited as LT.)

G. Birkhoff states in LT (p. 208, Example 4) that all join-endomorphisms $\Theta$ of any lattice $L$ form an $l$-semigroup, where the join of two join-endomorphisms $\Theta$ and $\Phi$ satisfies

$(\Theta \cup \Phi) x=\Theta x \cup \Phi x$

for all $x \in L$.

But this statement is not true in general; indeed, we shall show that there exists a lattice whose join-endomorphisms do not form an $l$ semigroup under the join stated.

We shall also deal with the following question proposed by $G$. Birkhoff in LT.

Problem 93. Is the lattice of all join-endomorphisms of an arbitrary lattice semi-modular?

We shall show that the answer is negative. ${ }^{1}$ Namely, restricting ourselves to finite lattices, we shall prove that there exists no lattice whose lattice of all join-endomorphisms is semi-modular and not distributive; furthermore, the lattice of all join-endomorphisms of any lattice $L$ is distributive if and only if $L$ is distributive.

We give also some generalizations of these results.

1. On the existence of the lattice of all join-endomorphisms. In what follows $L_{U}$ will denote the set of all join-endomorphisms of a lattice $L$, where the join of two elements $\Theta$ and $\Phi$ of $L_{U}$ is defined by

Received by the editors July 12, 1957; and, in revised form, November 22, 1957.

${ }^{1}$ This much was remarked by R. P. Dilworth in his review of I.T, Bull. Amer. Math. Soc. vol. 56 (1950) pp. 204-206. 
(2); the join operation gives rise to a partial ordering in $L_{U} ; \Theta \leqq \Phi$ if and only if $\Theta x \leqq \Phi x$ for all $x \in L$.

Firstly we show by an example that $L_{U}$ is in general not a lattice.

Let $V$ be the chain of all real numbers of the closed interval $[0,1]$ with the usual ordering. Let us consider in $V \cdot V$ (the cardinal product of $V$ by itself, in the sense of LT p. 7) the sublattice $L$ consisting of all elements of $V \cdot V$, with the exception of $(1,0) . L$ is actually a sublattice of $V \cdot V$, for $(1,0)$ is join- and meet-irreducible.

Let us consider the following mappings of $L$ into itself:

$$
\begin{aligned}
& \Theta=\{(1, y) \rightarrow(1,1) \text { if } y \neq 0 ;(x, y) \rightarrow(0,0) \text { if } x \neq 1\}, \\
& \Phi=\{(x, 0) \rightarrow(0,0) \text { if } x \neq 1 ;(x, y) \rightarrow(1, y) \text { if } y \neq 0\} .
\end{aligned}
$$

It is routine to check that $\Theta, \Phi \in L_{U}$. For all $\psi \in L_{U}$ satisfying $\psi \leqq \Theta$ and $\psi \leqq \Phi$ we have $\psi(x, y) \leqq \Theta(x, y)=(0,0)$ if $x \neq 1$, and for this reason $\psi(1, y)=\psi(1, z)$ holds for all $y \neq 0$ and $z \neq 0$. Let $\psi(1, y)$ $=(a, b)(y \neq 0)$. From $\Phi(1, y)=(1, y)$ and $\psi \leqq \Phi$ it follows that $(a, b)$ $\leqq(1, y)$ for all $y \neq 0$, that is $b=0$. Thus each $\psi$ is of the form $\psi$ $=\{(x, y) \rightarrow(0,0)$ if $x \neq 1 ;(1, y) \rightarrow(a, 0)$ if $y \neq 0\}$. Since among these $\psi$ there is clearly no greatest one, $a=1$ being impossible, it follows that $L_{U}$ is not a lattice.

In what follows we shall need the following sufficient condition for $L_{U}$ to be a lattice.

Theorem 1. If $L$ is a complete lattice, then $L_{U}$ is also a complete lattice.

Proof. Let $\Theta_{\alpha} \in L_{\cup}(\alpha \in A)$ and define $\Theta$ by $\Theta x=\mathrm{V}_{\alpha \in A} \Theta_{\alpha} x$. From the general associative law we get

$$
\begin{aligned}
\Theta x \cup \Theta y & =\bigvee_{\alpha \in A} \Theta_{\alpha} x \cup \underset{\alpha \in A}{\bigvee} \Theta_{\alpha} y=\bigvee_{\alpha \in A}\left(\Theta_{\alpha} x \cup \Theta_{\alpha} y\right) \\
& =\bigvee_{\alpha \in A} \Theta_{\alpha}(x \cup y)=\Theta(x \cup y)
\end{aligned}
$$

i.e., $\Theta \in L_{U}$. Clearly $\Theta \geqq \Theta_{\alpha}$; moreover if $\Phi \geqq \Theta_{\alpha}$ for all $\alpha \in A$, then $\Phi x \geqq \mathrm{~V}_{\alpha \in A} \Theta_{\alpha} x$ whence $\Phi \geqq \Theta$ and any subset of $L_{U}$ has a join. $L_{U}$ has a zero-element, for $L$ has a 0 and the mapping $x \rightarrow 0$ for all $x \in L$ is a join-endomorphism of $L$. Hence $L_{U}$ is a partly ordered set with zeroelement and complete joins, consequently $L_{U}$ is a complete lattice (LT, p. 49).

COROLlARY (ZACHER'S THEOREM). ${ }^{2}$ If $L$ is a finite lattice then all

${ }^{2}$ Giovanni Zacher, Sugli emiomorfismi superiori ed inferiori, Atti del Quarto Congresso dell'Unione Matematica Italiano, Taormina, vol. 2 (1951) pp. 251-252, and by the same author, with the same title in Rend. Accad. Sci. Fis. Mat. Napoli (4) vol. 19 (1952) pp. 45-56 (1953). 
join-endomorphisms of $L$ form a lattice.

2. Nondistributive lattices with finite bounded chains. ${ }^{3}$ If the lattice $L$ is not distributive, then it contains as a sublattice one of the lattices

$S$ : the elements of $S$ include $a, b, c, i, o ; a \cup b=b \cup c=c \cup a=i$, $a \cap b=b \cap c=c \cap a=o$;

$T$ : the elements of $T$ include $a, b, c, i, o ; a \cup c=b \cup c=i, a \cap c$ $=b \cap c=o, a \cap b=a$.

a. Let $L$ be a modular, but not distributive lattice with finite bounded chains. Then $L$ contains the lattice $S$ as a sublattice with the further condition that $a, b, c$ cover $o$ (LT, p. 134).

Let us consider in $L$ the following join-endomorphisms:

$$
\begin{aligned}
& \Theta=\{(a] \rightarrow o ; L-(a] \rightarrow a\}, \\
& \Phi=\{(o] \rightarrow o ;(b]-(o] \rightarrow b ;(c]-(o] \rightarrow c ; L-(b]-(c] \rightarrow i\}, \\
& \Psi=\{(o] \rightarrow o ;(b]-(o] \rightarrow b ; L-(b] \rightarrow i\}, \\
& \Omega=\{(i] \rightarrow o ; L-(i] \rightarrow a\} .
\end{aligned}
$$

Then $\Theta$ covers $\Omega$ and $\Theta \cup \Phi=\{(o] \rightarrow o ; L-(o) \rightarrow i\}$. Clearly $\Omega \cup \Phi$ $=\Phi<\Psi<\Theta \cup \Phi$ so that $L_{\cup}$ is not semi-modular.

b. If $L$ is a nonmodular lattice with finite bounded chains, then $L$ contains as a sublattice the lattice $T$. It is clear that there exist in $L$ an $x$ and $y$, such that $x$ covers $x \cap y$ and $x \cap y \neq y$. The mappings ( $\Im$ denotes a maximal ideal, such that $c \in \Im, a \in \Im$ )

$$
\begin{aligned}
& \Theta=\{\Im \rightarrow x \cap y ; L-\Im \rightarrow x\}, \\
& \Phi=\{(o] \rightarrow x \cap y ;(c]-(o] \rightarrow x ;(b]-(o] \rightarrow y ; L-(c]-(b] \rightarrow x \cup y\}, \\
& \Psi=\{(o] \rightarrow x \cap y ;(c]-(o] \rightarrow x ;(a]-(o] \rightarrow y ; \\
& \Omega=\{(i] \rightarrow x \cap y ; L-(i] \rightarrow x\}
\end{aligned}
$$

are join-endomorphisms and it may be easily checked that $\Theta$ covers $\Omega$, yet $\Theta \cup \Phi>\Psi>\Omega \cup \Phi=\Phi$, i.e. $L_{\cup}$ is not semi-modular. Thus we have the following

THEOREм 2. The lattice of all join-endomorphisms of a nondistributive lattice with finite bounded chains is not semi-modular.

3. The case of finite distributive lattices. Let $L$ be a distributive lattice with 0 and $I$, of finite length. It is known that $L$ is itself finite

${ }^{8}$ By a bounded chain we mean a chain with a least and a greatest element. ( $a$ ] denotes the ideal generated by $a$. 
and in $L$ every element is the join of join-irreducible elements. Conversely, if $L$ has exactly $k$ join-irreducible elements $a_{1}, a_{2}, \cdots, a_{k}$ then $\Theta \in L_{U}$ is completely determined by the elements $\boldsymbol{b}_{i}=\Theta a_{i}$ $(i=1,2, \cdots, k)$. Thus we may write $\Theta$ in the form $\Theta=\left(b_{1}, b_{2}, \cdots, b_{k}\right)$, where evidently $a_{i} \geqq a_{j}$ implies $b_{i} \geqq b_{j}$. Moreover

Lemma 1. If $a_{1}, \cdots, a_{k}$ are all the join-irreducible elements of $L$, and $b_{1}, \cdots, b_{k}$ are arbitrary in $L$, then a necessary and sufficient condition for the existence of a join-endomorphism $\Theta$ with $\Theta a_{i}=b_{i}$ $(i=1,2, \cdots, k)$ is the fulfilment of the condition: $a_{i} \geqq a_{j}$ implies $b_{i} \geqq b_{j}$.

Proof. Let us denote by $r(a)$ the set of all join-irreducible elements contained in $a$; then $a=\bigcup_{a_{i} \in r(a)} a_{i}$. We need only prove the sufficiency of the condition. If the $b_{i}$ satisfy the stated condition and we define $\Theta$ as $\Theta a=\bigcup_{a_{i} \in r(a)} \Theta a_{i}$, then we have to verify that $\Theta(a \cup b)$ $=\Theta a \cup \Theta b$ for all $a, b \in L$. Only $r(a) \bigvee r(b)=r(a \cup b)$ need be proved, where $\bigvee$ means the set-theoretical union. Evidently, $r(a) \bigvee r(b)$ $\subseteq r(a \cup b)$. On the other hand, if $x \in r(a \cup b)$ and $x \notin r(a), r(b)$, then $x \cap a<x, x \cap b<x$ and by the distributive law $x=(x \cap a) \cup(x \cap b)$ which is a contradiction; $\Theta a_{i}=b_{i}$ is obvious, completing the proof.

Lemma 2. Let $L$ be a finite distributive lattice and $k$ the number of its join-irreducible elements. Then $L_{\cup}$ may be imbedded as a sublattice in the cardinal product of $k$ lattices isomorphic with $L$.

Proof. Let $\Theta=\left(b_{1}, \cdots, b_{k}\right)$ and $\Phi=\left(c_{1}, \cdots, c_{k}\right)$ be join-endomorphisms of the lattices $L$. Let us consider $\Xi=\left(b_{1} \cap c_{1}, \cdots, b_{k} \cap c_{k}\right)$ and $\mathrm{H}=\left(b_{1} \cup c_{1}, \cdots, b_{k} \cup c_{k}\right)$. These are join-endomorphisms in view of Lemma 1. Evidently, $\Xi=\Theta \cap \Phi$ and $H=\Theta \cup \Phi$. Therefore the joinendomorphisms form a lattice isomorphic to a sublattice of the cardinal product of $k$ lattices isomorphic with $L$. q.e.d.

Consequently, $L_{U}$ is distributive, for it is a sublattice of a distributive lattice.

On the other hand, if $L_{U}$ is distributive, then so is $L$, because $L$ may be imbedded in $L_{\cup}$ by identifying $a \in L$ with the join-endomorphism $\Theta=(a, a, \cdots, a)$.

Summarizing the above statements, we arrive at

THEOREM 3. All join-endomorphisms of a finite lattice $L$ form a distributive lattice if and only if $L$ is distributive.

Obviously, our results may be generalized to lattices with 0 elements and finite bounded chains. 
LEMMA 2'. Let $L$ be a distributive lattice with 0 element and with finite bounded chains. $L_{\cup}$ may be imbedded in the discrete cardinal product of as many copies of $L$ as there exist join-irreducible elements in $L$.

Theorem $3^{\prime}$. Let $L$ be a lattice with 0 element and with finite bounded chains. All join-endomorphisms of $L$ form a distributive lattice if and only if $L$ is distributive.

BUDAPEST, HuNGARY

\section{LINEAR COMPLETENESS AND HYPERBOLIC TRIGONOMETRY}

\section{CURTIS M. FULTON}

In this paper we show the uniqueness of the relation between a segment and its angle of parallelism as derived from a model. Upon generalizing this relation hyperbolic trigonometry follows in a remarkably simple way.

To introduce proper terminology [5, pp. 11-28] let $\Sigma$ denote an axiom system, that is a certain set of axioms together with the undefined technical and logical or universal terms used to state the axioms. We define the terms interpretation and model in the usual fashion. It is useful to make a clear distinction between the following three concepts. (1) A $\Sigma$-statement is a meaningful expression, not necessarily true, in the technical and universal terms of $\Sigma$. (2) A $T$ - $\Sigma$-statement is a true $\Sigma$-statement in the sense of being logically derivable from $\Sigma$. (3) If $I$ denotes an interpretation of $\Sigma$, then an $I$ - $\Sigma$-statement is a $\Sigma$-statement holding for the model which results from the interpretation $I$.

For the purpose of this paper let $\Sigma$ be the postulate system of Hilbert [3, pp. 2-30] with the Euclidean axiom of parallelism replaced by the characteristic postulate of hyperbolic plane geometry $[6$, p. 66]. Some authors have used models to find $I$ - $\Sigma$-statements [ 1 , $\S 39-117 ; 2]$. Such a procedure, however, may be objectionable [1, $\$ 118]$. Conceivably an $I-\Sigma$-statement could be made which is not a $T$ - $\Sigma$-statement, but is merely a property of a particular model. In other words, it might be possible to find contradictory $I-\Sigma$-statements in two different models. Clearly, if this happens it indicates that our system $\Sigma$ is not complete [5, pp. 33-36]. Any $I-\Sigma$-statement that is not a $T-\Sigma$-statement would still be compatible with the axioms of $\Sigma$.

Presented to the Society April 19, 1958; received by the editors February 28, 1958. 